\title{
Abnormal resting-state functional connectivity within the default mode network subregions in male patients with obstructive sleep apnea
}

This article was published in the following Dove Press journal:

Neuropsychiatric Disease and Treatment

19 January 2016

Number of times this article has been viewed

\author{
Hai-Jun Li' \\ Xiao Nie' \\ Hong-Han Gong' \\ Wei Zhang ${ }^{2}$ \\ Si Nie' \\ De-Chang Peng' \\ 'Department of Radiology, \\ ${ }^{2}$ Department of Pneumology, The \\ First Affiliated Hospital of Nanchang \\ University, Nanchang, Jiangxi Province, \\ People's Republic of China
}

Background and objective: Abnormal resting-state functional connectivity (rs-FC) between the central executive network and the default mode network (DMN) in patients with obstructive sleep apnea (OSA) has been reported. However, the effect of OSA on rs-FC within the DMN subregions remains uncertain. This study was designed to investigate whether the rs-FC within the DMN subregions was disrupted and determine its relationship with clinical symptoms in patients with OSA.

Methods: Forty male patients newly diagnosed with severe OSA and 40 male education- and age-matched good sleepers (GSs) underwent functional magnetic resonance imaging (fMRI) examinations and clinical and neuropsychologic assessments. Seed-based region of interest rs-FC method was used to analyze the connectivity between each pair of subregions within the DMN, including the medial prefrontal cortex (MPFC), posterior cingulate cortex (PCC), hippocampus formation (HF), inferior parietal cortices (IPC), and medial temporal lobe (MTL). The abnormal rs-FC strength within the DMN subregions was correlated with clinical and neuropsychologic assessments using Pearson correlation analysis in patients with OSA.

Results: Compared with GSs, patients with OSA had significantly decreased rs-FC between the right HF and the PCC, MPFC, and left MTL. However, patients with OSA had significantly increased rs-FC between the MPFC and left and right IPC, and between the left IPC and right IPC. The rs-FC between the right HF and left MTL was positively correlated with rapid eye movement ( $r=0.335, P=0.035$ ). The rs-FC between the PCC and right HF was negatively correlated with delayed memory $(r=-0.338, P=0.033)$.

Conclusion: OSA selectively impairs the rs-FC between right HF and PCC, MPFC, and left MTL within the DMN subregions, and provides an imaging indicator for assessment of cognitive dysfunction in OSA patients.

Keywords: obstructive sleep apnea, functional connectivity, default mode network, functional MRI, cognitive impairment

\section{Introduction}

Obstructive sleep apnea (OSA) is a common sleep-related breathing disorder caused by repeated complete or partial collapse of the upper airway during sleep, resulting in intermittent hypoxia ( $\mathrm{IH})$, intermittent hypercapnia, and sleep fragmentation. ${ }^{1}$ Based on the results of populations studies, it has been found that OSA affects approximately $5.7 \%-6.0 \%$ of middle-aged men and $2.4 \%-4.0 \%$ of middle-aged women. ${ }^{2,3}$ OSA has been shown to increase daytime sleepiness, road traffic accidents, stroke, hypertension, coronary artery disease, congestive heart failure, insulin resistance, and cardiovascular mortality..$^{4-8}$ The common cognitive impairments, including impaired memory, learning, and attention have been commonly observed in patients with OSA, because
Correspondence: De-Chang Peng Affiliated Hospital of Nanchang University, No 17, Yongwai Zheng Street, Donghu District, Nanchang 330006, Jiangxi Province, People's Republic of China

Tel +86 79l 88694457

Email pengdcdoctor@163.com 
of both sleep disturbances and hypoxemia. ${ }^{9}{ }^{10}$ However, the underlying neural mechanisms remain unclear.

Structural and functional neuroimaging techniques have evolved and have been used to increase our understanding of neurocognitive processes and structural brain differences. Previous voxel-based morphometry (VBM) studies have reported conflicting results in patients with OSA, ${ }^{11-18}$ and failed to fully explain the differences in the pathophysiology or severity of disease. For example, one study failed to identify any regions of gray matter (GM) reduction. ${ }^{14}$ Another study showed widespread loss of more than 20 foci of GM concentration. ${ }^{17}$ Impaired hippocampus was a relatively consistent finding across different neuroimaging techniques in previous studies. ${ }^{12,13,16,17,19}$ The hippocampus region is closely associated with neural processing of memory. ${ }^{20}$ Prilipko et al ${ }^{21}$ found that patients with OSA had a significant inactivation in the medial temporal regions within the default mode network (DMN) and a significant activation in the right ventral frontoparietal network during the tasks. In addition, Sweet et $\mathrm{al}^{22}$ found a deactivation in the posterior cingulate and right postcentral gyrus within the DMN during continuous positive airway pressure (CPAP) withdrawal during working memory tasks using functional magnetic resonance imaging (fMRI) in patients with OSA. Magnetic resonance spectroscopy is a useful neuroimaging tool to measure changes in either the concentration or distribution of chemical substances. Bartlett et $\mathrm{al}^{23}$ found an elevated $N$-acetylaspartate (NAA)-to-creatine (Cr) ratio (NAA/Cr) and lowered $\mathrm{Cr}$ levels in the left hippocampus area, which were associated with neurocognitive performance and OSA severity. O'Donoghue et $\mathrm{al}^{24}$ found a decreased frontal NAA-to-choline (Cho) ratio (NAA/Cho) and hippocampus $\mathrm{Cho} / \mathrm{Cr}$ ratio in patients with OSA, which persisted after CPAP treatment. However, in the recent years, resting-state fMRI (rs-fMRI)-based functional connectivity (FC) has been widely used for objective analysis of the brain's functional connectome. ${ }^{25,26}$ Resting-state FC-based region of interest (ROI) has been regarded as a reliable technology. ${ }^{27,28}$ It has been increasingly used in studies of OSA and other sleep disorders. ${ }^{29,30}$ The DMN comprises multiple dissociated subregions, including medial prefrontal cortex (MPFC), posterior cingulate cortex (PCC), bilateral hippocampus formation (HF), bilateral inferior parietal cortices (IPC), and bilateral medial temporal lobe (MTL). Many studies have suggested that the DMN might be associated with the collection and evaluation of information, ${ }^{31}$ self-referential mental activity, ${ }^{32}$ extraction of episodic memory, ${ }^{33}$ emotion and anxiety, ${ }^{34,35}$ and mind wandering or daydreaming. ${ }^{36}$
Our previous study found that patients with OSA showed local abnormal spontaneous activity including part of the DMN using amplitude of low-frequency fluctuation (ALFF) and regional homogeneity (ReHo) method. ${ }^{37,38}$ These findings suggest that the DMN is associated with sleep disorder. Recent studies have confirmed abnormal rs-FC within the DMN subregions in various diseases, including multiple sclerosis, hepatic encephalopathy, and sleep deprivation. ${ }^{39-41}$ Although numerous neuroimaging studies have been conducted to identify structural and functional impairments in the brains of OSA patients, the effect of OSA on the intrinsic DMN node connectivity is still unknown. In this fMRI study, we hypothesized the rs-FC within the DMN subregions was disrupted in OSA patients.

To test the hypothesis, we first investigated the rs-FC patterns within DMN subregions using seed-based ROI correlation analyses methods in patients with OSA and in good sleepers (GSs). Next, we compared the intergroup differences in the rs-FC within the DMN subregion in patients with OSA and those with GSs. Finally, we evaluated the relationships between the altered rs-FC within the DMN subregions and disease severity and neuropsychologic performance in patients with OSA.

\section{Materials and methods Subjects}

Forty male patients newly diagnosed with severe OSA and 40 male education- and age-matched GSs were included from the Sleep Monitoring Room of the Respiratory Department of The First Affiliated Hospital of Nanchang University. The inclusion and exclusion criteria for the patients with OSA and GSs were consistent with our previous studies. ${ }^{37,38}$ Inclusion criteria for the patients with OSA were male sex, age older than 22 years but younger than 60 years, and an apneahypopnea index (AHI) greater than 30 . Inclusion criteria for GSs were male sex, age older than 22 years but younger than 60 years, and an AHI less than 5. The exclusion criteria for both patients with OSA and for GSs were 1) sleep disorders other than OSA, such as insomnia; 2) hypertension, diabetes, and respiratory and heart diseases; 3 ) neurodegenerative diseases, head injury, epilepsy, psychosis, depressive disorder, and hypothyroidism; 4) history of cerebrovascular disease; 5) alcohol or illicit drug abuse; 6) a structural lesion on brain MRI; and 7) MRI contraindications, such as claustrophobia, metallic implants, or devices in the body. Each candidate underwent a detailed clinical interview, sleep questionnaire, and overnight polysomnography (PSG). Informed written consent was obtained from all the subjects. The study was 
approved by the Medical Research Ethics Committee of The First Affiliated Hospital of Nanchang University.

\section{Overnight PSG}

The day before sleep studies, all GSs and patients with OSA were required to abstain from drinking caffeinated or alcoholic beverages. Overnight PSG monitoring was performed on all patients with OSA and GSs using the Respironics LE-Series physiological monitoring system (Alice 5 LE, Respironics, Orlando, FL, USA). PSG was recorded from approximately $10 \mathrm{pm}$ to $6 \mathrm{am}$ the next day. The electroencephalogram (EEG), electrooculogram (EOG), electrocardiogram, chin electromyogram (EMG), oral and nasal airflow, thoracic and abdominal movements, body position, oxygen saturation $\left(\mathrm{SaO}_{2}\right)$, and snoring were recorded. According to the American Academy of Sleep Medicine (AASM) guidelines ${ }^{42}$ the EEG derivations (from frontal, central, occipital regions: F4/M1, C4/M1, O2/M1; and back up derivations: F3/M2, C3/M2, and O1/M2), EMG (located in the three chin electrodes and the middle of the right anterior tibialis), and EOG (located in the cornea and retina) were recorded. Sleep latency, total sleep time, sleep efficiency, sleep stages, arousal, and respiratory events were also recorded. According to the AASM manual, an obstructive apnea was defined as a reduction in airflow $\geq 90 \%$ lasting at least 10 seconds and associated with persistent respiratory effort; hypopnea was defined as a reduction in airflow $\geq 30 \%$ lasting at least 10 seconds and accompanied with a $4 \%$ or greater oxygen desaturation. ${ }^{42}$ The AHI was calculated as the average of the total number of apnea and hypopnea events experienced per hour of sleep. The arousal index (AI) was computed as the mean number of EEG arousals per hour of sleep.

\section{Neuropsychological assessments}

All patients with OSA and GSs were evaluated with a selfreported sleep questionnaire for excessive daytime sleepiness using Epworth sleepiness scale (ESS), which asks the subject to rate his or her probability of falling asleep on a scale of increasing probability from 0 to 3 for eight different situations. ${ }^{43} \mathrm{~A}$ score equal to or greater than 10 demonstrates excessive daytime sleepiness in OSA patients. Cognitive function was evaluated using the Montreal Cognitive Assessment (MoCA), including naming, executive function, calculation, attention, language, memory, abstraction, and orientation, with a total MoCA score lower than 26 indicating cognitive impairment. ${ }^{44}$ If the length of subjects' education was less than 12 years, one point was added to the total score, as education deviation adjustment. ${ }^{44}$

\section{MRI data acquisition}

Imaging was performed on a $3.0 \mathrm{~T}$ MRI system with eightchannel head coil (Siemens, Munich, Germany). Earplugs were used to minimize scanner noise, and foam pads were used to reduce head movements. First, conventional T1-weighted imaging and $\mathrm{T} 2$-weighted imaging were performed. Then, the rs-fMRI images were collected using an echo planar imaging (EPI) sequence with the following parameters: repetition time $(\mathrm{TR})=2,000 \mathrm{~ms}$, echo time $(\mathrm{TE})=30 \mathrm{~ms}$, thickness $=4.0 \mathrm{~mm}$, gap $=1.2 \mathrm{~mm}$, field of view $(\mathrm{FOV})=230 \mathrm{~mm} \times 230 \mathrm{~mm}$, matrix $=64 \times 64$, and flip angle $=90^{\circ}$. Each brain volume comprised 30 axial slices, and each functional run consisted of 240 volumes. During rs-fMRI scans, all subjects were instructed to keep their eyes closed, to be still, to think of nothing in particular, and not to fall asleep. Finally, high-resolution three-dimensional T1-weighted images were obtained by a brain volume sequence $(\mathrm{TR}=1,900 \mathrm{~ms}, \mathrm{TE}=2.26 \mathrm{~ms}$, thickness $=1.0 \mathrm{~mm}$, gap $=0.5 \mathrm{~mm}$, FOV $=250 \mathrm{~mm} \times 250 \mathrm{~mm}$, matrix $=256 \times 256$, flip angle $=9^{\circ}, 176$ sagittal slices).

\section{fMRI data preprocessing}

Functional data were checked by MRIcro software (www. MRIcro.com) to exclude defective data. The rs-fMRI data were analyzed using the Data Processing Assistant for Resting-State fMRI Advanced Edition (DPARSFA; http:// rfmri.org/DPARSFA) on the basis of MATLAB2010a (Mathworks, Natick, MA, USA). The first ten volumes of each subject were discarded due to the signal reaching equilibrium and the participants adapted to the scanning noise. The remaining 230 volumes were corrected for delay in acquisition time between different slices and corrected for geometrical displacements according to the estimated head movement. The Friston six-head motion parameters were computed by estimating translation in each direction and the angular rotation on each axis for each volume based on recent work showing that higher order models were more effective in removing head motion effects. ${ }^{45,46}$ Each subject showed a maximum displacement of less than $1.5 \mathrm{~mm}$ in any cardinal direction $(x, y, z)$ and a maximum spin $(x, y, z)$ of less than $1.5^{\circ}$. A multiple regression method was performed to remove possible sources of artifacts, including six estimated motion parameters, ventricular and white matter regions, and global signal. ${ }^{47,48}$ Subsequently, all functional data were spatially normalized to the Montreal Neurological Institute (MNI) EPI template, and resampled to $3 \times 3 \times 3 \mathrm{~mm}^{3}$ voxels and smoothed with a $6 \mathrm{~mm}$ full width at half maximum. Finally, a temporal filter $(0.01-0.08 \mathrm{~Hz})$ was applied to reduce the effect of lowfrequency drift and high-frequency noise. 


\section{Definition of DMN subregions}

In previous research, ${ }^{38,49,50}$ we defined eight core subregions within the DMN: PCC (MNI coordinates: 0, -53 , 26), MPFC (MNI coordinates: 0, 52, -6), left HF (MNI coordinates: $-24,-22,-20)$, right HF (MNI coordinates: 24, -20, -22), left MTL (MNI coordinates: -43, -74, 28), right MTL (MNI coordinates: 47, -57, 20), left IPC (MNI coordinates: -29, 26, -28), and right IPC (MNI coordinates: $29,26,-28)$. Next, the average time series of a $6 \mathrm{~mm}$ sphere centered at the peak coordinate of each subregion was extracted from each patient with OSA and GS, and the rs-FC map of each pairwise subregion was obtained.

\section{rs-FC analysis of DMN subregions}

For each patient with OSA and GS, Pearson correlation coefficients between the mean time series of each pairwise DMN subregion were computed and converted to $z$-values using Fisher's $r$-to- $z$ transformation to improve the normality.

\section{Statistical analysis}

The demographic and clinical data were compared between the two groups using independent sample $t$-test, which was conducted with Statistical Package for the Social Sciences version 19.0 (SPSS, Chicago, IL, USA). Differences were considered significant when $P<0.05$.

For the rs-FC within the DMN subregions, first, a single sample $t$-test was performed on individual $z$-value map of the 28 pairs of DMN subregion connectivities within each group. Then, we analyzed intergroup differences after adding the body mass index (BMI) and age as covariates of no interest using two independent sample $t$-tests. A corrected significance level of $P<0.05$, using the false-positive adjustment, was used to determine the statistical significance.

We investigated the relationship between clinical and neuropsychologic assessments and the altered rs-FC value of pairwise subregions within the DMN in patients with OSA. Each significant rs-FC value was extracted and used for ROI-based correlation analyses with clinical variables using linear correlation analysis. $P<0.05$ was deemed statistically significant.

\section{Results}

\section{Demographic and clinical data}

There were no significant differences between patients with OSA and GSs in age and years of education $(P>0.05$; Table 1). The patients with OSA had significantly higher scores for BMI $(t=7.43, P<0.001)$, AHI $(t=17.20, P<0.001)$, $\mathrm{SaO}_{2}<90 \%(t=8.03, P<0.001)$, AI $(t=8.09, P<0.001)$, and ESS $(t=12.42, P<0.001)$, but a significantly lower score for rapid eye movement $(\mathrm{REM})$ sleep $(t=-8.03, P<0.001)$ and MoCA $(t=-5.09, P=0.036)$ when compared with GSs.

\section{rs-FC of pairwise subregions within the DMN between patients with OSA and GSs}

In the two groups, all pairwise DMN subregions showed strong correlation (Figure 1). Compared with GSs, patients with OSA displayed significantly decreased rs-FC between

Table I Demographic and clinical data of patients with OSA and GSs

\begin{tabular}{|c|c|c|c|c|c|c|}
\hline \multirow[t]{2}{*}{ Characteristic } & \multicolumn{2}{|c|}{ OSA patients $(N=40)$} & \multicolumn{2}{|c|}{ GSs $(N=40)$} & \multirow[t]{2}{*}{ t-value } & \multirow[t]{2}{*}{$P$-value } \\
\hline & Mean & SD & Mean & SD & & \\
\hline Age, years & 38.6 & 8.1 & 39.3 & 7.50 & -0.39 & 0.700 \\
\hline Education, years & 11.8 & 3.2 & I I.0 & 3.50 & 0.96 & 0.342 \\
\hline $\mathrm{BMI}, \mathrm{kg} / \mathrm{m}^{2}$ & 27.8 & 3.4 & 23.1 & 2.10 & 7.43 & $<0.001$ \\
\hline Sleep onset latency, min & 10.2 & 8.9 & 18.3 & 9.50 & -2.97 & 0.005 \\
\hline Total sleep time, min & 386.2 & 80.1 & 398.3 & 20.20 & -0.93 & 0.358 \\
\hline Sleep efficiency, \% & 78.6 & 5.3 & 91.3 & 5.80 & -4.15 & $<0.001$ \\
\hline AHI/hour & 59.5 & 20.9 & 2.5 & 1.30 & 17.20 & $<0.001$ \\
\hline Stage I, \% & 30.4 & 18.8 & 10.0 & 3.80 & 6.73 & $<0.001$ \\
\hline Stage $2, \%$ & 38.4 & I5.| & 38.7 & 6.60 & 0.92 & 0.362 \\
\hline Stage $3+4, \%$ & 17.0 & 19.3 & 23.2 & 7.90 & -3.97 & $<0.001$ \\
\hline REM, \% & 7.2 & 8.0 & 21.3 & 7.70 & -8.03 & $<0.001$ \\
\hline $\mathrm{SaO}_{2}<90 \%$ & 28.0 & 21.9 & 0.2 & 0.20 & 8.03 & $<0.001$ \\
\hline Nadir $\mathrm{SaO}_{2}$ & 66.0 & 12.4 & 93.6 & 4.72 & 10.57 & $<0.001$ \\
\hline $\mathrm{Al} /$ hour & 42.5 & 23.4 & II.7 & 2.90 & 8.09 & $<0.001$ \\
\hline ESS & 12.1 & 3.9 & 3.3 & 2.30 & 12.42 & $<0.001$ \\
\hline MoCA & 25.1 & 2.5 & 27.8 & 1.50 & -5.90 & $<0.001$ \\
\hline
\end{tabular}

Abbreviations: OSA, obstructive sleep apnea; GSs, good sleepers; N, number; SD, standard deviation; BMI, body mass index; AHI, apnea-hypopnea index; REM, rapid eye movement; $\mathrm{SaO}_{2}$, oxygen saturation; $\mathrm{Al}$, arousal index; $\mathrm{SaO}_{2}<90 \%$, percentage of total sleep time spent at oxygen saturation less than $90 \%$; ESS, Epworth sleepiness scale; MoCA, Montreal Cognitive Assessment. 

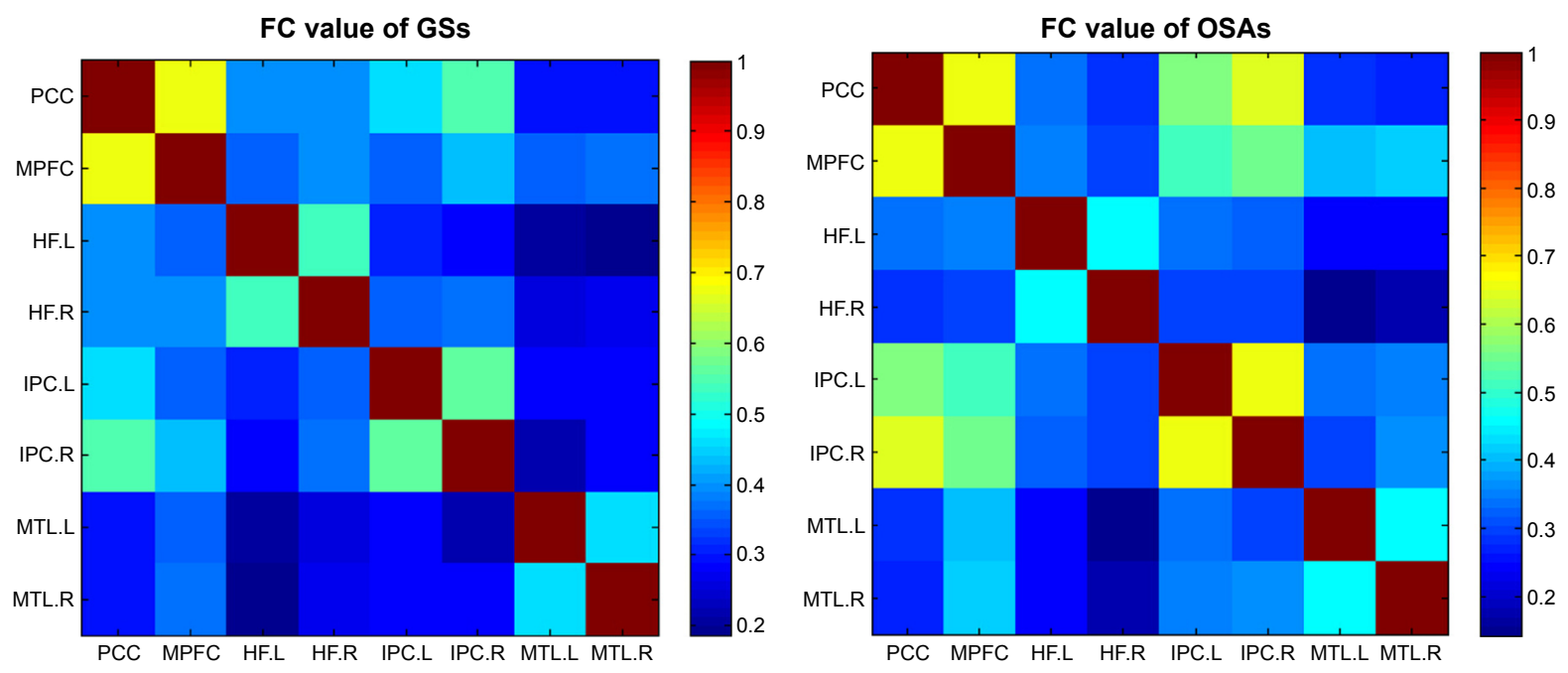

Figure I Correlation matrix of the average time series of pairwise subregions within the DMN.

Notes: The pictures represent the FC results of the 28 pairs of DMN subregions in GSs and patients with OSA respectively by one-sample $t$-test. Different colors stand for different connection coefficients.

Abbreviations: FC, functional connectivity; DMN, default mode network; GSs, good sleepers; OSAs, patients with obstructive sleep apnea; MPFC, medial prefrontal cortex; PCC, posterior cingulate cortex; HF, hippocampus formation; MTL, medial temporal lobe; IPC, inferior parietal cortices; R, right; L, left.

the right HF and the PCC, MPFC, and left MTL. However, patients with OSA showed significantly increased rs-FC between left IPC and right IPC, and between the MPFC and left and right IPC (Table 2, Figure 2). Abnormal connection coefficients were extracted (Figure 3).

\section{Correlations}

In patients with OSA, the AHI score was significantly positively correlated with BMI $(r=0.418, P=0.007)$, ESS $(r=0.430, P=0.006), \mathrm{AI}(r=0.732, P<0.001)$, and negatively correlated with nadir $\mathrm{SaO}_{2}(r=-0.499, P=0.001)$ and mean $\mathrm{SaO}_{2}(r=-0.467, P=0.002)$. BMI displayed significantly positive correlation with $\mathrm{AI}(r=0.546, P<0.001)$, and negative correlation with the nadir $\mathrm{SaO}_{2}(r=-0.613, P<0.001)$.

Table 2 Abnormal rs-FC of pairwise subregions within the DMN between patients with OSA and GSs

\begin{tabular}{|c|c|c|c|}
\hline ROI I & ROI 2 & $t$-value ${ }^{a}$ & $P$-value \\
\hline $\begin{array}{l}\text { Right hippocampal } \\
\text { formation }\end{array}$ & $\begin{array}{l}\text { Posterior } \\
\text { cingulate cortex }\end{array}$ & -2.32 & 0.023 \\
\hline $\begin{array}{l}\text { Right hippocampal } \\
\text { formation }\end{array}$ & $\begin{array}{l}\text { Medial prefrontal } \\
\text { cortex }\end{array}$ & -2.10 & 0.039 \\
\hline $\begin{array}{l}\text { Right hippocampal } \\
\text { formation }\end{array}$ & $\begin{array}{l}\text { Left medial } \\
\text { temporal lobe }\end{array}$ & -2.05 & 0.043 \\
\hline $\begin{array}{l}\text { Medial prefrontal } \\
\text { cortex }\end{array}$ & $\begin{array}{l}\text { Left inferior } \\
\text { parietal cortex }\end{array}$ & 2.49 & 0.015 \\
\hline $\begin{array}{l}\text { Medial prefrontal } \\
\text { cortex }\end{array}$ & $\begin{array}{l}\text { Right inferior } \\
\text { parietal cortex }\end{array}$ & 2.59 & 0.012 \\
\hline $\begin{array}{l}\text { Left inferior } \\
\text { parietal cortex }\end{array}$ & $\begin{array}{l}\text { Right inferior } \\
\text { parietal cortex }\end{array}$ & 2.70 & 0.009 \\
\hline
\end{tabular}

Note: ${ }^{a} \mathrm{~A}$ negative/positive $t$-value represents a decrease/increase of FC.

Abbreviations: rs-FC, resting-state functional connectivity; OSA, obstructive sleep apnea; GSs, good sleepers; ROI, region of interest; DMN, default mode network.
In patients with OSA, significant positive correlations were found between the rs-FC of the right HF with left MTL and the REM\% ( $r=0.335, P=0.035)$. Significant negative correlations were found between the rs-FC of the PCC with right HF and delayed memory ( $r=-0.338, P=0.033$; Figure 4).

\section{Discussion}

Abnormal rs-FC between the right anterior insula and the two anticorrelated cognitive-related networks (the central executive network and the DMN) has been reported in patients with OSA..$^{29}$ However, to the best of our knowledge, our study is the first to explore group differences between specific subregions within the DMN using seed-based ROI rs-FC method in patients with OSA. We found that patients with OSA displayed decreased rs-FC between the right HF and the PCC, MPFC, and left MTL, and concomitant increase in rs-FC between left IPC and right IPC and between the MPFC and left and right IPC, compared with GSs. Furthermore, we found that the rs-FC between the right HF and left MTL was positively correlated with the REM\%, and the rs-FC between the PCC and right HF was negatively correlated with delayed memory. Our findings indicated aberrant rs-FC within the DMN subregions in patients with OSA during the resting state.

Obesity, age, and male sex were the main risk factors for OSA. Patients with OSA had significantly higher BMI compared with GSs in our study. Sforza et al ${ }^{51}$ found that patients with OSA showed significant sex differences in clinical symptoms, and females with OSA exhibited a lower AHI, less severe hypoxia, and greater peripheral fat mass, 

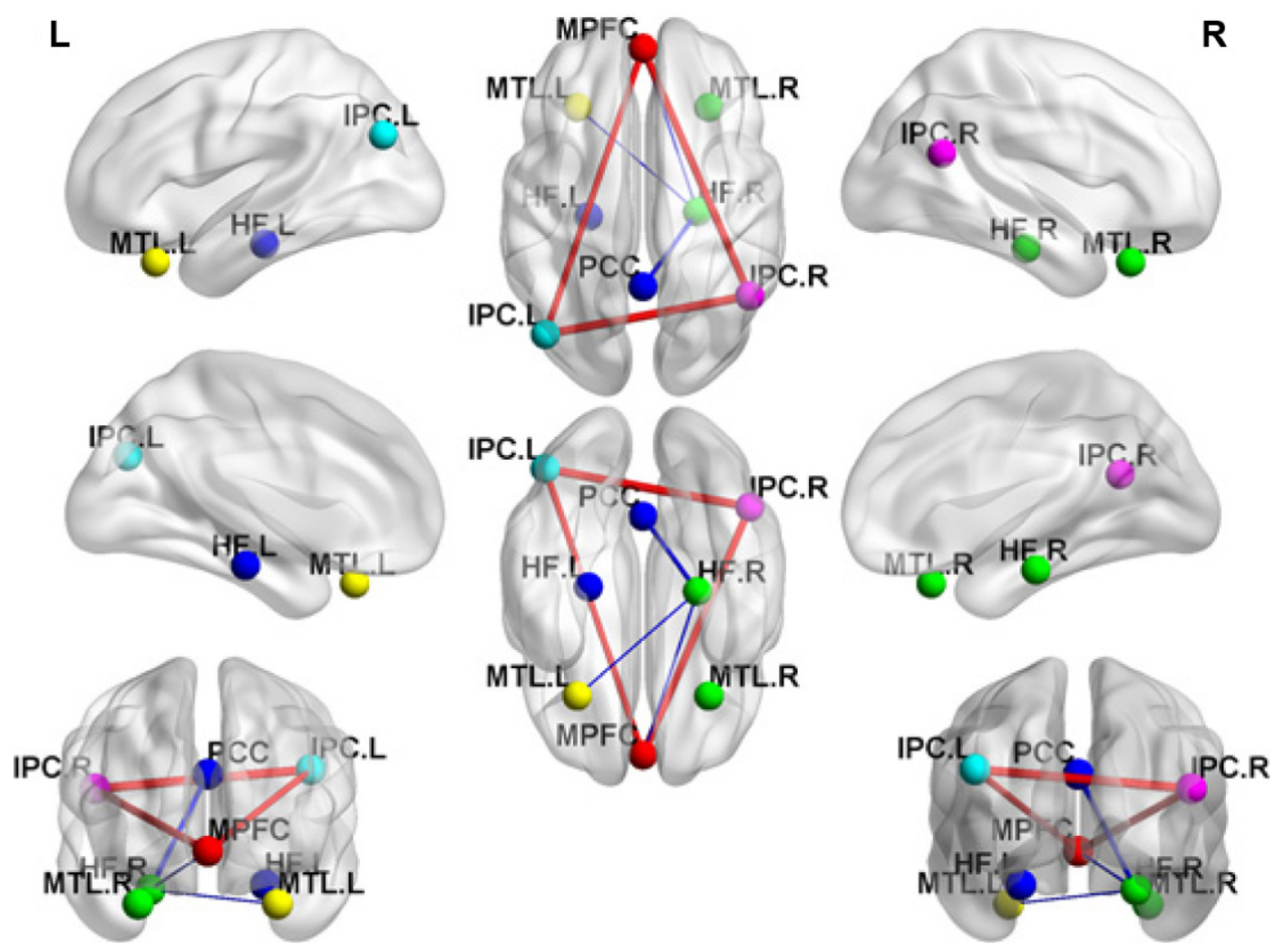

Figure 2 Compared with GSs, patients with OSA showed abnormal rs-FC of pairwise subregions within the DMN.

Notes: The blue line stands for decreased rs-FC within the DMN. The red line stands for increased rs-FC within the DMN. Undirected edges correspond to the significance of differences ( $t$-values).

Abbreviations: GSs, good sleepers; OSA, obstructive sleep apnea; rs-FC, resting-state functional connectivity; DMN, default mode network; MPFC, medial prefrontal cortex; PCC, posterior cingulate cortex; HF, hippocampus formation; MTL, medial temporal lobe; IPC, inferior parietal cortices; R, right; L, left.

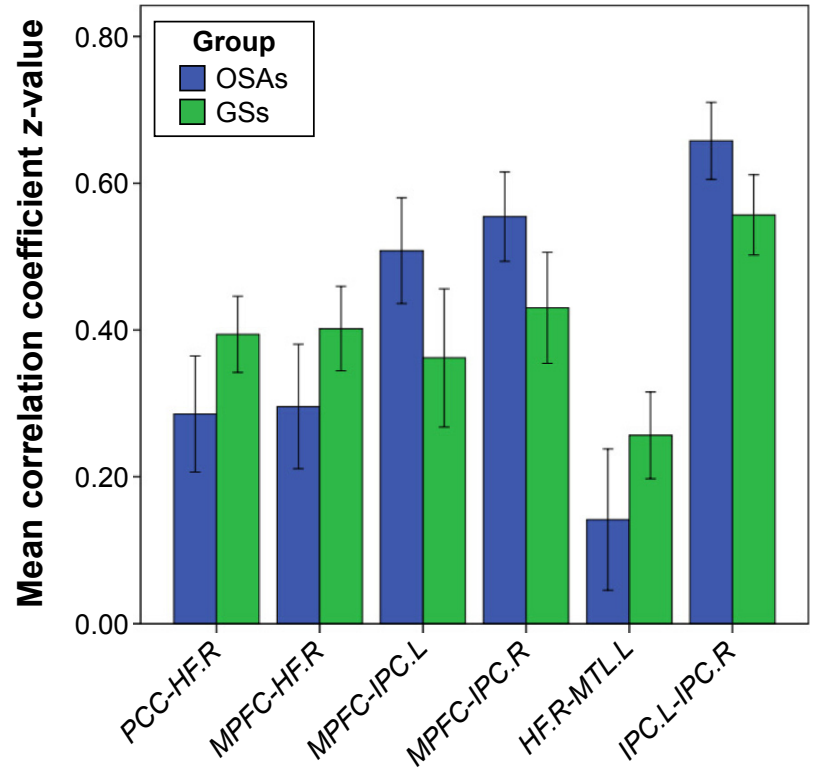

Functional connectivity region-to-region

Figure 3 Mean correlation coefficient $z$-values of abnormal rs-FC of pairwise subregions within the DMN.

Note: The right stapeldiagram presents mean correlation coefficient $z$-values between patients with OSA and GSs.

Abbreviations: GSs, good sleepers; OSAs, patients with obstructive sleep apnea; rs-FC, resting-state functional connectivity; MPFC, medial prefrontal cortex; PCC, posterior cingulate cortex; HF, hippocampus formation; MTL, medial temporal lobe; IPC, inferior parietal cortices; R, right; L, left; DMN, default mode network. and also frequently reported anxiety and depression. Previous studies had demonstrated that obesity and sex differences likely influence the resting-state brain activity. ${ }^{52,53}$ Controlling for the BMI and sex differences weakened the statistical differences in the rs-FC between patients with OSA and GSs. To confirm that our rs-FC findings were mainly due to OSA rather than obesity or sex differences, we compared the intergroup differences in rs-FC within the DMN subregions with BMI and age as covariates, and only adult male OSA patients were recruited. We still observed significant rs-FC between the right HF and PCC, MPFC, and left MTL with the same statistical threshold, suggesting that the reduced rs-FC was related to OSA rather than obesity.

Although the exact neural mechanisms underlying reduced rs-FC between the PCC and right HF were unknown, they may be related to structural or functional impairment in the PCC or right HF. Greicius et $\mathrm{al}^{54}$ found that the PCC plays a pivotal role in the $\mathrm{DMN}$, which has a strong anatomical relationship with the rest of the DMN. Moreover, the PCC displayed a strong reciprocal connection and other related memory structures, such as the HF, and played a critical role in episodic memory retrieval. ${ }^{55}$ Previous studies found that hippocampal 
A

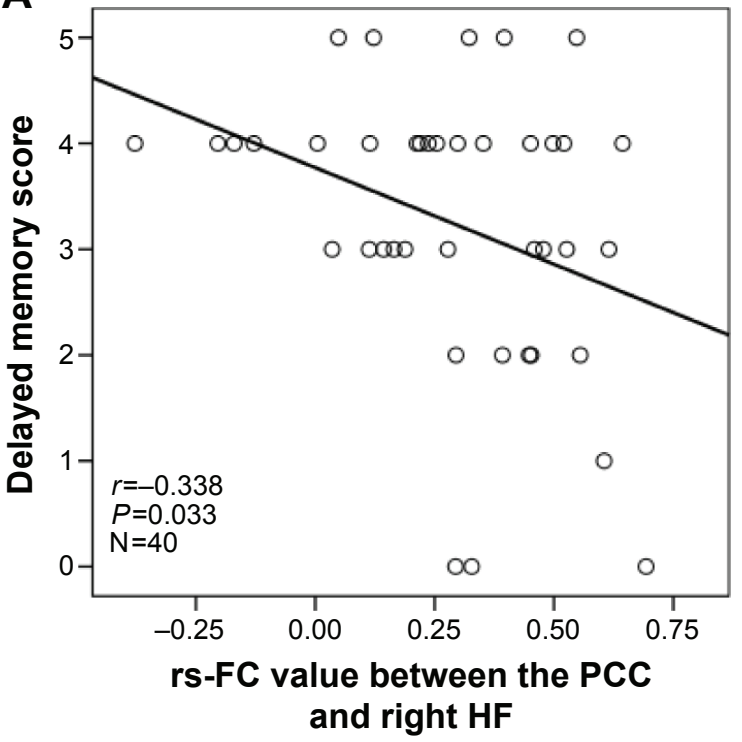

B

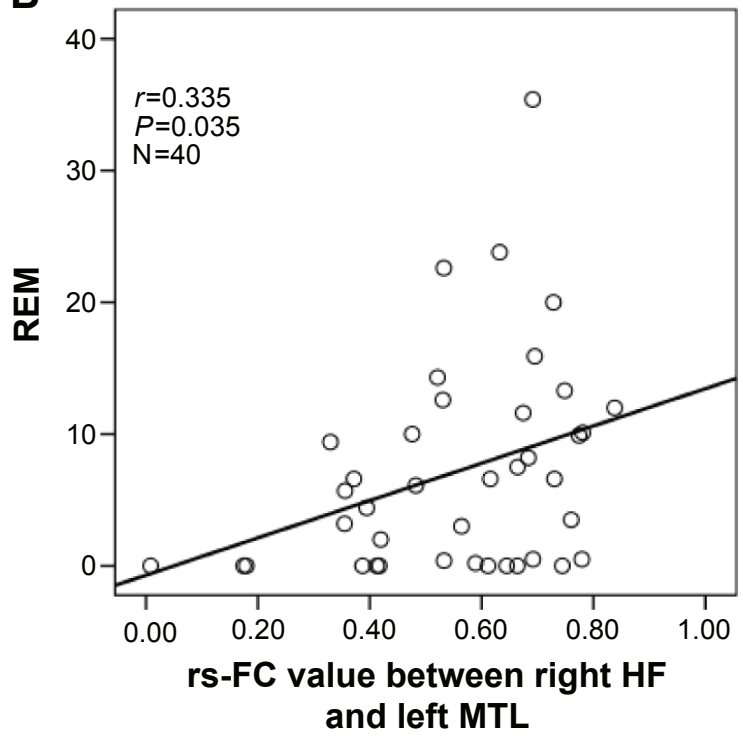

Figure 4 Scatter plots demonstrate significant correlations between the rs-FC of pairwise subregions within the DMN and OSA severity and neuropsychologic scores in patients with OSA.

Notes: The rs-FC between the PCC and right HF displays significant negative correlation with delayed memory score (A). The rs-FC between the right HF and left MTL demonstrates significant positive correlation with REM (B).

Abbreviations: OSA, obstructive sleep apnea; rs-FC, resting-state functional connectivity; REM, rapid eye movement; PCC, posterior cingulate cortex; HF, hippocampus formation; MTL, medial temporal lobe; DMN, default mode network.

structural or functional impairments were relatively consistent across different neuroimaging techniques in OSA. ${ }^{12,13,16,17,19}$ A few studies have showed abnormal deactivation and metabolism in the PCC in patients with OSA. ${ }^{22,56}$ Our previous study found that patients with OSA showed significantly lower ALFF value in the PCC, and displayed a significant positive correlations with the nadir $\mathrm{SaO}_{2}$, suggesting that $\mathrm{IH}$ may be an important factor underlying PCC dysfunction in OSA. ${ }^{38}$ Prilipko et $\mathrm{l}^{57}$ found that patients with OSA displayed abnormal inactivation in the DMN during working memory tasks and significant positive correlation with behavioral performance, suggesting that inhibition of the resting activity in the DMN plays an important role in cognitive impairment. A previous study has shown greater ipsilateral HF atrophy and reduced FC between the ipsilateral HF and the PCC in patients with temporal lobe epilepsy. ${ }^{58}$ Consistent with these results, our study found that patients with OSA displayed significantly decreased rs-FC between the PCC and right HF. Furthermore, the rs-FC between the PCC and right HF displayed significant negative correlation with delayed memory score, suggesting that reduced rs-FC between the PCC and right HF might be an imaging indicator of cognitive impairment in OSA.

Previous studies have shown that the MTL subsystem, composed of the MPFC, posterior IPC, retrosplenial cortex, parahippocampal cortex, and $\mathrm{HF}$, showed increased activity in participants during episodic decisions and that the MPFC was the most important nodal hub. ${ }^{59}$ Zhang et $\mathrm{al}^{60}$ found that patients with OSA demonstrated decreased rs-FC and reduced GMV in the MPFC using independent component analysis and VBM methods, respectively, suggesting that the structural and functional impairments in the MPFC might be a candidate mechanism for the cognitive and emotional deficits. A previous study showed reduced FC between the MPFC and the HF in patients with depression and schizophrenia. ${ }^{61}$ The prefrontal cortex and HF were vulnerable to impairment by sleep disruption and $\mathrm{IH}^{62,63}$ and decreased task-related activation in the MPFC and hippocampal atrophy were frequently reported in patients with OSA. ${ }^{64,65}$ The hippocampus and MPFC play distinct and important roles in emotional processing, spatial memory, and navigation. ${ }^{66,67}$ Our study found that OSA was associated with significantly decreased rs-FC between the right HF and the MPFC, probably related to $\mathrm{IH}$ and sleep fragmentation, respectively, resulting in neuronal deficits in the HF and the MPFC. ${ }^{68,69}$

A previous study has found that cerebrovascular reactivity of the MTL was negatively correlated with duration of nocturnal hypoxemia, suggesting a possible pathophysiological mechanism for hippocampal injury. ${ }^{70}$ The volume of the HF and the MTL atrophy were also reported, which is associated with memory impairment. ${ }^{71}$ Consistent with the previous study, our study found significantly decreased rs-FC between right $\mathrm{HF}$ and left MTL in patients with OSA. We also found that the rs-FC between the right HF and left MTL was positively correlated with REM sleep. REM sleep has 
been shown to be necessary for cortical synaptic plasticity and for the acquisition of spatial and nonspatial memory. ${ }^{72}$ Fragmented REM sleep resulting from REM-sleep-specific respiratory events and related arousal might have resulted in low REM sleep amount in patients with OSA. Low REM sleep may play an important role in the HF and the MTL for memory impairment in patients with OSA.

The IPC is implicated in attention processing, ${ }^{73}$ and is unintentionally or obligatorily occupied in the recall, consolidation, and retrieval of episodic memory information. ${ }^{74,75}$ Previous studies described overactivation of the right IPC during a two-back verbal working memory after acute withdrawal from positive airway pressure treatment in OSA. ${ }^{76}$ Similarly, Ayalon et $\mathrm{al}^{77}$ found that patients with OSA had increased brain activation in the left IPC, and was negatively correlated with recall during verbal learning task, suggesting that patients with OSA displayed an adaptive compensatory recruitment response. Consistent with the findings, our study found that patients with OSA had significantly increased rs-FC between left and right IPC, and between the MPFC and left and right IPC. These brain regions may be involved in an adaptive compensatory response for cognitive impairment in patients with OSA.

\section{Limitations}

Several limitations should be considered when interpreting our results. First, considering the convention of sample collection and homogeneity, we only recruited adult males with severe OSA. Females, children, and mild-to-moderate OSA cases need to be investigated in future studies. Previous observational studies demonstrated that children with OSA had increased executive dysfunction and depression symptoms compared with the normal children. ${ }^{78,79}$ However, the underlying neural mechanism is not clear. Second, we only observed the rs-FC within the DMN subregions. Diffusion tensor imaging technology enables elucidation of the anatomical basis of abnormal rs-FC within the DMN subregions. Finally, we only studied the abnormal rs-FC within the DMN subregions before treatment in OSA. Additional investigations are needed to explore whether the abnormal rs-FC within the DMN subregions can be improved after treatment.

\section{Conclusion}

In this study, we investigated alterations in connectivity of pairwise subregions within the DMN in patients with OSA using seed-based ROI rs-FC methods. We found that OSA selectively impairs the rs-FC between right $\mathrm{HF}$ and PCC, MPFC, and left MTL within the DMN subregions, thereby providing an imaging indicator for the assessment of cognitive dysfunction in patients with OSA.

\section{Acknowledgments}

This work was supported by the Natural Science Foundation of China (grant number 81560285), Jiangxi Provincial Department of Natural Science Foundation Project (grant number 20132BAB205100), Jiangxi Provincial Department of Science and Technology Support Program (grant numbers 20132BBG70061 and 20141BBG70026), and Jiangxi Provincial Department of Graduate Innovation Foundation (grant number YC2015-S082).

\section{Disclosure}

None of the authors have received payment or services from a third party (government, commercial, private foundation, etc) for any aspect of the submitted work at any time. The authors report no conflicts of interest in this work.

\section{References}

1. Jordan AS, McSharry DG, Malhotra A. Adult obstructive sleep apnoea. Lancet. 2014;383(9918):736-747.

2. Liu JH, Wei CZ, Huang LY, et al. Prevalence of signs and symptoms suggestive of obstructive sleep apnea syndrome in Guangxi, China. Sleep Breath. 2014;18(2):375-382.

3. Franklin KA, Lindberg E. Obstructive sleep apnea is a common disorder in the population - a review on the epidemiology of sleep apnea. J Thorac Dis. 2015;7(8):1311-1322.

4. Teran-Santos J, Jimenez-Gomez A, Cordero-Guevara J. The association between sleep apnea and the risk of traffic accidents. Cooperative Group Burgos-Santander. N Engl J Med. 1999;340(11):847-851.

5. Oldenburg O, Lamp B, Faber L, et al. Sleep-disordered breathing in patients with symptomatic heart failure: a contemporary study of prevalence in and characteristics of 700 patients. Eur J Heart Fail. 2007;9(3):251-257.

6. Strohl KP, Brown DB, Collop N, et al. ATS Ad Hoc Committee on Sleep Apnea, Sleepiness, and Driving Risk in Noncommercial Drivers. An official American Thoracic Society clinical practice guideline: sleep apnea, sleepiness, and driving risk in noncommercial drivers. $\mathrm{Am}$ J Respir Crit Care Med. 2013;187(11):1259-1266.

7. Foster GD, Sanders MH, Millman R, et al. Obstructive sleep apnea among obese patients with type 2 diabetes. Diabetes Care. 2009;32(6): 1017-1019.

8. Saunamaki T, Jehkonen M. Depression and anxiety in obstructive sleep apnea syndrome: a review. Acta Neurol Scand. 2007;116(5): 277-288.

9. Engleman HM, Douglas NJ. Sleep 4: sleepiness, cognitive function, and quality of life in obstructive sleep apnoea/hypopnoea syndrome. Thorax. 2004;59(7):618-622.

10. Xu M, Yang Y, Zhang JJ. Levels of neuroglobin in serum and neurocognitive impairments in Chinese patients with obstructive sleep apnea. Sleep Breath. 2013;17(2):573-582.

11. Kamba M, Inoue $Y$, Higami S, et al. Cerebral metabolic impairment in patients with obstructive sleep apnea: an independent association of obstructive sleep apnea with white matter change. J Neurol Neurosurg Psychiatry. 2001;71(3):334-339.

12. Macey PM, Henderson LA, Macey KE, et al. Brain morphology associated with obstructive sleep apnea. Am J Respir Crit Care Med. 2002;166(10): $1382-1387$. 
13. Morrell MJ, McRobbie DW, Quest RA, et al. Changes in brain morphology associated with obstructive sleep apnea. Sleep Med. 2003; 4(5):451-454.

14. O'Donoghue FJ, Briellmann RS, Rochford PD, et al. Cerebral structural changes in severe obstructive sleep apnea. Am J Respir Crit Care Med. 2005;171(10):1185-1190.

15. Macey PM, Kumar R, Woo MA, et al. Brain structural changes in obstructive sleep apnea. Sleep. 2008;31(7):967-977.

16. Canessa N, Castronovo V, Cappa SF, et al. Obstructive sleep apnea: brain structural changes and neurocognitive function before and after treatment. Am J Respir Crit Care Med. 2011;183(10):1419-1426.

17. Joo EY, Tae WS, Lee MJ, et al. Reduced brain gray matter concentration in patients with obstructive sleep apnea syndrome. Sleep. 2010; 33(2):235-241.

18. Macey PM, Kumar R, Yan-Go FL, et al. Sex differences in white matter alterations accompanying obstructive sleep apnea. Sleep. 2012; 35(12):1603-1613.

19. Torelli F, Moscufo N, Garreffa G, et al. Cognitive profile and brain morphological changes in obstructive sleep apnea. Neuroimage. 2011; 54(2):787-793.

20. Burgess N, Maguire EA, O'Keefe J. The human hippocampus and spatial and episodic memory. Neuron. 2002;35(4):625-641.

21. Prilipko O, Huynh N, Schwartz S, et al. The effects of CPAP treatment on task positive and default mode networks in obstructive sleep apnea patients: an fMRI study. PLoS One. 2012;7(12):e47433.

22. Sweet LH, Jerskey BA, Aloia MS. Default network response to a working memory challenge after withdrawal of continuous positive airway pressure treatment for obstructive sleep apnea. Brain Imaging Behav. 2010;4(2):155-163.

23. Bartlett DJ, Rae C, Thompson CH, et al. Hippocampal area metabolites relate to severity and cognitive function in obstructive sleep apnea. Sleep Med. 2004;5(6):593-596.

24. O'Donoghue FJ, Wellard RM, Rochford PD, et al. Magnetic resonance spectroscopy and neurocognitive dysfunction in obstructive sleep apnea before and after CPAP treatment. Sleep. 2012;35(1):41-48.

25. Friston KJ. Functional and effective connectivity: a review. Brain Connect. 2011;1(1):13-36.

26. Van Dijk KR, Hedden T, Venkataraman A, et al. Intrinsic functional connectivity as a tool for human connectomics: theory, properties, and optimization. J Neurophysiol. 2010;103(1):297-321.

27. Fox MD, Snyder AZ, Vincent JL, et al. The human brain is intrinsically organized into dynamic, anticorrelated functional networks. Proc Natl Acad Sci U S A. 2005;102(27):9673-9678.

28. Fransson P. Spontaneous low-frequency BOLD signal fluctuations:an fMRI investigation of resting-state default mode of brain function hypothesis. Hum Brain Mapp. 2005;26(1):15-29.

29. Zhang Q, Qin W, He XX, et al. Functional disconnection of the right anterior insula in obstructive sleep apnea. Sleep Med. 2015;16(9): 1062-1070.

30. Yeo BT, Tandi J, Chee MW. Functional connectivity during rested wakefulness predicts vulnerability to sleep deprivation. Neuroimage. 2015;17(111):147-158.

31. Gusnard DA, Raichle ME, Raichle ME. Searching for a baseline: functional imaging and the resting human brain. Nat Rev Neurosci. 2001; 2(10):685-694.

32. Gusnard DA, Akbudak E, Shulman GL, et al. Medial prefrontal cortex and self-referential mental activity: relation to a default mode of brain function. Proc Natl Acad Sci U S A. 2001;98(7):4259-4264.

33. Cabeza R, Dolcos F, Graham R, et al. Similarities and differences in the neural correlates of episodic memory retrieval and working memory. Neuroimage. 2002;16(2):317-330.

34. Simpson JR Jr, Drevets WC, Snyder AZ, et al. Emotion-induced changes in human medial prefrontal cortex: II. During anticipatory anxiety. Proc Natl Acad Sci U S A. 2001;98(2):688-693.

35. Simpson JR Jr, Snyder AZ, Gusnard DA, et al. Emotion-induced changes in human medial prefrontal cortex: I. During cognitive task performance. Proc Natl Acad Sci U S A. 2001;98(2):683-687.
36. Mason MF, Norton MI, Van Horn JD, et al. Wandering minds: the default network and stimulus-independent thought. Science. 2007; 315(5810):393-395.

37. Peng DC, Dai XJ, Gong HH, et al. Altered intrinsic regional brain activity in male patients with severe obstructive sleep apnea: a restingstate functional magnetic resonance imaging study. Neuropsychiatr Dis Treat. 2014;10:1819-1826.

38. Li HJ, Dai XJ, Gong HH, et al. Aberrant spontaneous low-frequency brain activity in male patients with severe obstructive sleep apnea revealed by resting-state functional MRI. Neuropsychiatr Dis Treat. 2015;11:207-214.

39. Zhou F, Zhuang Y, Gong H, et al. Altered inter-subregion connectivity of the default mode network in relapsing remitting multiple sclerosis: a functional and structural connectivity study. PLoS One. 2014;9(7):e101198.

40. Qi RF, Zhang LJ, Xu Q, et al. Abnormal functional connectivity within the default mode network in patients with HBV-related cirrhosis without hepatic encephalopathy revealed by resting-state functional MRI. Brain Res. 2014;1576:73-80.

41. Dai XJ, Liu CL, Gong HH, et al. Long-term sleep deprivation decreases the default spontaneous activity and connectivity pattern in healthy male subjects: a resting-state fMRI study. Neuropsychiatr Dis Treat. 2015;11:761-772.

42. Iber C, Ancoli-Israel S, Chesson A, et al; for the American Academy of Sleep Medicine. The AASM Manual for the Scoring of Sleep and Associated Events: Rules, Terminology and Technical Specifications. 1st ed. Westchester, IL: American Academy of Sleep Medicine; 2007.

43. Billings ME, Rosen CL, Auckley D, et al. Psychometric performance and responsiveness of the functional outcomes of sleep questionnaire and sleep apnea quality of life instrument in a randomized trial: the HomePAP study. Sleep. 2014;37(12):2017-2024.

44. Nasreddine ZS, Phillips NA, Bédirian V, et al. The Montreal Cognitive Assessment, MoCA: a brief screening tool for mild Cognitive impairment. J Am Geriatr Soc. 2005;53:695-699.

45. Satterthwaite TD, Elliott MA, Gerraty RT, et al. An improved framework for confound regression and filtering for control of motion artifact in the preprocessing of resting-state functional connectivity data. Neuroimage. 2013;64:240-256.

46. Yan CG, Cheung B, Kelly C, et al. A comprehensive assessment of regional variation in the impact of head micromovements on functional connectomics. Neuroimage. 2013;76:183-201.

47. Guo W, Jiang J, Xiao C, et al. Decreased resting-state interhemispheric functional connectivity in unaffected siblings of schizophrenia patients. Schizophr Res. 2014;152(1):170-175.

48. Saad ZS, Gotts SJ, Murphy K, et al. Trouble at rest: how correlation patterns and group differences become distorted after global signal regression. Brain Connect. 2012;2(1):25-32.

49. Hedden T, Van Dijk KR, Becker JA, et al. Disruption of functional connectivity in clinically normal older adults harboring amyloid burden. J Neurosci. 2009;29(40):12686-12694.

50. Zhang D, Raichle ME. Disease and the brain's dark energy. Nat Rev Neurol. 2010;6(1):15-28.

51. Sforza E, Chouchou F, Collet P, et al. Sex differences in obstructive sleep apnoea in an elderly French population. Eur Respir J. 2011;37(5): $1137-1143$

52. Dai XJ, Gong HH, Wang YX, et al. Gender differences in brain regional homogeneity of healthy subjects after normal sleep and after sleep deprivation: a resting-state fMRI study. Sleep Med. 2012;13(6):720-727.

53. Black WR, Lepping RJ, Bruce AS, et al. Tonic hyper-connectivity of reward neurocircuitry in obese children. Obesity (Silver Spring). 2014; 22(7):1590-1593.

54. Greicius MD, Supekar K, Menon V, et al. Resting-state functional connectivity reflects structural connectivity in the default mode network. Cereb Cortex. 2009;19(1):72-78.

55. Torta DM, Cauda F. Different functions in the cingulate cortex, a metaanalytic connectivity modeling study. Neuroimage. 2011;56(4): 2157-2172. 
56. Yaouhi K, Bertran F, Clochon P, et al. A combined neuropsychological and brain imaging study of obstructive sleep apnea. J Sleep Res. 2009; $18: 36-48$.

57. Prilipko O, Huynh N, Schwartz S, et al. Task positive and default mode networks during a parametric working memory task in obstructive sleep apnea patients and healthy controls. Sleep. 2011;34(3):293-301.

58. Kemmotsu N, Kucukboyaci NE, Cheng CE, et al. Alterations in functional connectivity between hippocampus and prefrontal cortex as a correlate of depressive symptoms in temporal lobe epilepsy. Epilepsy Behav. 2013;29(3):552-559.

59. Andrews-Hanna JR, Reidler JS, Sepulcre J, et al. Functional-anatomic fractionation of the brain's default network. Neuron. 2010;65(4): $550-562$.

60. Zhang Q, Wang D, Qin W, et al. Altered resting-state brain activity in obstructive sleep apnea. Sleep. 2013;36(5):651-659.

61. Genzel L, Dresler M, Cornu M, et al. Medial prefrontal-hippocampal connectivity and motor memory consolidation in depression and schizophrenia. Biol Psychiatry. 2015;77(2):177-186.

62. Beebe DW, Gozal D. Obstructive sleep apnea and the prefrontal cortex: towards a comprehensive model; linking nocturnal upper airway obstruction to daytime cognitive and behavioral deficits. J Sleep Res. 2002;11(1):1-16.

63. Gozal D, Daniel JM, Dohanich GP. Behavioral and anatomical correlates of chronic episodic hypoxia during sleep in the rat. J Neurosci. 2001;21(7):2442-2450.

64. Zhang X, Ma L, Li S, et al. A functional MRI evaluation of frontal dysfunction in patients with severe obstructive sleep apnea. Sleep Med. 2011;12(4):335-340.

65. Morrell MJ, Jackson ML, Twigg GL, et al. Changes in brain morphology in patients with obstructive sleep apnoea. Thorax. 2010;65(10): 908-914.

66. Cross RL, Kumar R, Macey PM, et al. Neural alterations and depressive symptoms in obstructive sleep apnea patients. Sleep. 2008;31(8): 1103-1109.

67. Maguire EA, Gadian DG, Johnsrude IS, et al. Navigation-related structural change in the hippocampi of taxi drivers. Proc Natl Acad Sci US A. 2000;97(8):4398-4403.
68. Morrell MJ, Twigg G. Neural consequences of sleep disordered breathing: the role of intermittent hypoxia. Adv Exp Med Biol. 2006;588: 75-88.

69. Row BW, Liu R, Xu W, et al. Intermittent hypoxia is associated with oxidative stress and spatial learning deficits in the rat. Am J Respir Crit Care Med. 2003;167:1548-1553.

70. Prilipko O, Huynh N, Thomason ME, et al. An fMRI study of cerebrovascular reactivity and perfusion in obstructive sleep apnea patients before and after CPAP treatment. Sleep Med. 2014;15(8):892-898.

71. Daulatzai MA. Evidence of neurodegeneration in obstructive sleep apnea: Relationship between obstructive sleep apnea and cognitive dysfunction in the elderly. J Neurosci Res. 2015;24:1-17.

72. Romcy-Pereira R, Pavlides C. Distinct modulatory effects of sleep on the maintenance of hippocampal and medial prefrontal cortex LTP. Eur J Neurosci. 2004;20(12):3453-3462.

73. Supekar K, Uddin LQ, Prater K, et al. Development of functional and structural connectivity within the default mode network in young children. Neuroimage. 2010;52(1):290-301.

74. Foster DJ, Wilson MA. Reverse replay of behavioural sequences in hippocampal place cells during the awake state. Nature. 2006; 440(7084):680-683.

75. Wig GS, Grafton ST, Demos KE, et al. Medial temporal lobe BOLD activity at rest predicts individual differences in memory ability in healthy young adults. Proc Natl Acad Sci U S A. 2008;105(47): $18555-18560$

76. Aloia MS, Sweet LH, Jerskey BA, et al.Treatment effects on brain activity during a working memory task in obstructive sleep apnea. J Sleep Res. 2009;18(4):404-410.

77. Ayalon L, Ancoli-Israel S, Klemfuss Z, et al. Increased brain activation during verbal learning in obstructive sleep apnea. Neuroimage. 2006;31(4):1817-1825.

78. Esposito M, Antinolfi L, Gallai B, et al. Executive dysfunction in children affected by obstructive sleep apnea syndrome: an observational study. Neuropsychiatr Dis Treat. 2013;9:1087-1094.

79. Carotenuto M, Esposito M, Parisi L, et al. Depressive symptoms and childhood sleep apnea syndrome. Neuropsychiatr Dis Treat. 2012;8:369-373.
Neuropsychiatric Disease and Treatment

\section{Publish your work in this journal}

Neuropsychiatric Disease and Treatment is an international, peerreviewed journal of clinical therapeutics and pharmacology focusing on concise rapid reporting of clinical or pre-clinical studies on a range of neuropsychiatric and neurological disorders. This journal is indexed on PubMed Central, the 'PsycINFO' database and CAS,

\section{Dovepress}

and is the official journal of The International Neuropsychiatric Association (INA). The manuscript management system is completely online and includes a very quick and fair peer-review system, which is all easy to use. Visit http://www.dovepress.com/testimonials.php to read real quotes from published authors. 